in the liver, heart, kidney, lung, and spleen of treated mice, which suggests that treatment with I-Lys could represent a specific and safe therapeutic strategy against caspase-3-downregulated tumors. The authors also demonstrated that low doses of I-Lys, incapable of inducing apoptosis as a single agent, synergistically increased sensitivity to chemotherapy-induced cell death in multidrug-resistant cancer cells. Despite these promising findings, more rigorous preclinical studies are still needed to establish whether I-Lys could be used in human cancer therapy. To turn I-Lys into a pharmacological agent for clinical use will require more in-depth analysis of its pharmacokinetics, bioavailability, and tolerability. Nonetheless, the present study by Lin et al. (4) provides a strategy for precision medicine, namely the biomarker of caspase- 3 deficiency, and suggests a novel approach to treat such tumors.

\section{Acknowledgments}

The secretarial assistance of Courtney Hoover is greatly appreciated.

Address correspondence to: Gregory J. Gores, College of Medicine, Mayo Clinic, 200 First Street SW, Rochester, Minnesota 55905, USA. Phone: 507.284.0686; Fax: 507.284.0762;

E-mail: gores.gregory@mayo.edu.

1. Devarajan E, et al. Down-regulation of caspase 3 in breast cancer: a possible mechanism for chemoresistance. Oncogene. 2002;21(57):8843-8851.

2. Twiddy D, Cohen GM, Macfarlane M, Cain K. Caspase-7 is directly activated by the approximately $700-\mathrm{kDa}$ apoptosome complex and is released as a stable XIAP-caspase-7 approximately $200-\mathrm{kDa}$ complex. J Biol Chem. 2006;281(7):3876-3888.

3. Scott FL, Denault JB, Riedl SJ, Shin H, Renatus M, Salvesen GS. XIAP inhibits caspase- 3 and -7 using two binding sites: evolutionarily conserved mechanism of IAPs. EMBO J. 2005;24(3):645-655

4. Lin Y-F, et al. Targeting the XIAP/caspase-7 complex selectively kills caspase-3-deficient malignancies. J Clin Invest. 2013;123(9):3861-3875.

5. Lamkanfi M, Festjens N, Declercq W, Vanden Ber- ghe T, Vandenabeele P. Caspases in cell survival, proliferation and differentiation. Cell Death Differ. 2007;14(1):44-55

6. Hornle M, Peters N, Thayaparasingham B, Vorsmann H, Kashkar H, Kulms D. Caspase-3 cleaves XIAP in a positive feedback loop to sensitize melanoma cells to TRAIL-induced apoptosis. Oncogene. 2011;30(5):575-587.

7. Fulda S, Vucic D. Targeting IAP proteins for therapeutic intervention in cancer. Nat Rev Drug Discov. 2012;11(2):109-124.

8. Janicke RU, Sprengart ML, Wati MR, Porter AG. Caspase- 3 is required for DNA fragmentation and morphological changes associated with apoptosis. J Biol Chem. 1998;273(16):9357-9360.

9. Quintavalle $\mathrm{C}$, et al. Effect of miR-21 and miR$30 \mathrm{~b} / \mathrm{c}$ on TRAIL-induced apoptosis in glioma cells [published online ahead of print September 10, 2012]. Oncogene doi: 10.1038/onc.2012.410.

10. Zhang C, Zhao J, Deng H. 17beta-Estradiol up-regulates miR-155 expression and reduces TP53INP1 expression in MCF-7 breast cancer cells. Mol Cell Biochem. 2013;379(1-2):201-211.

11. Zheng SR, Guo GL, Zhai Q, Zou ZY, Zhang W. Effects of miR-155 antisense oligonucleotide on breast carcinoma cell line MDA-MB-157 and implanted tumors. Asian Pac J Cancer P. 2013; 14(4):2361-2366

\title{
Donor-associated malignancy in kidney transplant patients
}

\author{
Cai-Bin Cui and David A. Gerber
}

Department of Surgery, University of North Carolina at Chapel Hill School of Medicine, Chapel Hill, North Carolina, USA.

\begin{abstract}
Skin cancer cells with donor genotype have been identified in allogeneic transplant patients; however, the donor contribution to the recipient's epithelial malignancy remains to be established. In this issue of the JCI, Verneuil et al. provide the first evidence for donor contribution to the malignant epithelium of skin squamous cell carcinoma in a kidney transplant recipient. This case report may have important implications for cancer research and clinical care of long-surviving kidney transplant patients.
\end{abstract}

\section{Introduction}

Kidney transplantation is the preferred treatment for end-stage kidney disease due to improved patient survival and quality of life as well as lower treatment costs compared with dialysis (1). However, as transplant recipients live longer and a greater number of older donors are used (2), longterm complications, such as cancer as a leading cause of death in patients with a functioning graft, will begin to emerge. This outcome can predominantly be attributed to the immunosuppression required

Conflict of interest: The authors have declared that no conflict of interest exists.

Citation for this article: J Clin Invest. 2013; 123(9):3708-3709. doi:10.1172/JCI70438. to avoid rejection of the transplanted organ (3). The incidence of skin cancer is increased in transplant recipients, especially in kidney transplant recipients (KTRs), for which squamous cell carcinoma (SCC) is most common (4-6).

In most cases, skin SCC originates from the recipient's epithelium, but donor cells from transplanted kidney could also be a source. While more than 64 cases of donor cell leukemia have been reported in bone marrow transplant patients (7), only one skin basal cell carcinoma (BCC) has been previously reported as being donor associated in allogeneic KTR (8). In this issue of the JCI, Verneuil et al. provide the first convincing evidence for a direct donor contribution to the malignant epithelium of skin SCC in a KTR (9).

\section{Skin carcinogenesis}

In contrast to the prevalence of BCC in the general population, skin SCC is predominant in KTRs $(4,10)$. Skin SCC is a fully differentiated type of skin carcinoma originating mainly from epithelium and is the most common skin cancer in transplant recipients, occurring 65 to 250 times as frequently as in the general population $(4,6)$. Skin carcinogenesis in KTRs is a complex, incompletely understood process. Multiple oncogenic events include gene mutations (e.g., TP53, which encodes $\mathrm{p} 53$, and KRAS) introduced by UV radiation $(3,11)$, viral infection, or germline inheritance. More than 250 independent germline TP53 mutations have been discovered (12). Such mutations are typically associated with Li-Fraumeni syndrome, a clinically and genetically heterogeneous autosomal-dominant inherited cancer syndrome $(12,13)$. Immunosuppressive medications prevent the immune system from removing the mutant cancer cells, which - combined with the deleterious 
synergistic effects of $\operatorname{UV}(8,11)$ and other events - may initiate and/or promote the process of skin carcinogenesis $(3,13,14)$.

\section{Donor-associated versus de novo malignancy in transplant recipients}

The majority of skin SCCs in KTRs originate from the recipient's skin epithelium, but donor cells from the transplanted kidney can also serve as a source. Verneuil et al. (9) reviewed 21 skin SCCs from KTRs; in one patient, they identified a skin SCC with donor genotype, but not the recipient's. They confirmed that the microdis-

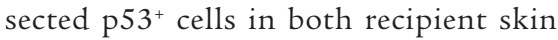
SCC and donor renal tubules had the same mitochondrial DNA-high-resolution melting patterns in all three markers, but were different from the recipient's DNA. In addition, they found that the skin SCC carried the same TP53 c.524G>A mutation (p.Arg175His, also known as rs28934578) as in donor renal tubule $\mathrm{p} 53^{+}$cells, but not in the normal recipient cells. This germline mutation in TP53 was different from the common UV-induced tandem CC>TT mutation. The authors conclude that the recipient's skin SCC originated from donor renal tubule cells and provide convincing evidence for direct donor contribution to the malignant epithelium of skin SCC in a KTR. They also identified a KRAS mutation in skin SCC, but not in donor cells, which indicates that the KRAS mutation is a new somatic mutation. The patient skin SCC was located in a UV-exposed area, and the combination of KRAS and TP53 mutations could be a key to initiation and/or promotion of skin epithelial carcinogenesis.

Although it is unclear how donor renal cells migrate to skin and form a tumor, donor-associated malignancy (DAM) should perhaps be approached differently than de novo malignancy (DNM) in a transplant recipient. Because donor cells migrate to new foreign sites, such as recipient skin, it is important to determine how they adapt to the new microenvironmental niche, what effects result from the new interactions, and the effects of donor cells on tumorigenesis. Similar to donor cell leukemia (15), there is undoubtedly some mechanistic overlap between the development of DAM and DNM. In the pathogenesis of DAM, it is important to consider that its cause is multifactorial in nature. Factors intrinsic to the cell and external signaling cues from the niche determine a normal versus neoplastic fate for the transplanted donor cells. Continued research to char- acterize DAM will help to understand the dynamic equilibrium between both normal and cancer stem cells and the skin microenvironment $(7,15)$. These interactions could help explain why the $\mathrm{p} 53^{+}$renal tubule cells with the same TP53 mutation in the KTR described by Verneuil et al. never formed a renal tumor (9).

\section{Unanswered questions}

Cancer, especially skin SCC, is a leading cause of mortality and morbidity in longsurviving KTRs. Future research should address what can be done to reduce/prevent DNM and DAM in KTRs. Should a cancer risk genetic test be included in transplant recipient and/or donor organ screening? For recipient screenings, biochemical, but not genetic, cancer screenings have been included in the screening guidelines for KTRs (16). Genetic factors are increasingly recognized to play important roles in tumorigenesis. Furthermore, genetic cancer risk screening of transplant recipients is potentially reasonable, since the cost and turnaround time for genetic testing is rapidly improving (17). For donor organ screening, current screening does not include genetic testing for cancer risk gene mutations. It is unclear whether the current report of DAM (9) should prompt a change in the screening approach. The likelihood of performing genetic testing on donor organs is remote at this point, due to the extremely low prevalence of DAM, the long turnaround time, the high cost associated with the genetic tests, and the ever-increasing clinical shortage of donor organs.

Other factors, including organ preservation techniques, contribute to donor cell migration to other organs. Longer cold ischemia time increases the apoptosis of the renal tubules, and more cells and cell debris are shed into the bloodstream. Reducing shedding of donor tissue cells into circulation will reduce homing of donor cells to recipient skin, thereby reducing the possibilities of carcinogenesis (18).

In summary, the increase in long-term survival for solid organ transplant recipients and the knowledge of germline mutations and their association with oncogenesis brings us to a new clinical decision point. While the majority of post-transplant malignancies are likely to remain a de novo malignancy, we may be at the beginning of a time when we can risk assess the possibility of DAM development in a transplant recipient.

\section{Acknowledgments}

We thank Chengwen Li (University of North Carolina at Chapel Hill) and XinHai Pei (University of Miami) for their critical comments.

Address correspondence to: David A. Gerber, Department of Surgery, University of North Carolina at Chapel Hill School of Medicine, Chapel Hill, North Carolina 27599, USA. Phone: 919.966.8008; Fax: 919.966.6308; E-mail: david_gerber@med.unc.edu.

1. First MR, Gerber DA, Hariharan S, Kaufman DB, Shapiro R. Posttransplant diabetes mellitus in kidney allograft recipients: incidence, risk factors, and management. Transplantation. 2002;73(3):379-386.

2. Gallagher MP, et al. Long-term cancer risk of immunosuppressive regimens after kidney transplantation. J Am Soc Nephrol. 2010;21(5):852-858.

3. Vajdic CM, van Leeuwen MT. Cancer incidence and risk factors after solid organ transplantation. Int $J$ Cancer. 2009;125(8):1747-1754.

4. Euvrard S, Kanitakis J, Claudy A. Skin cancers after organ transplantation. $N$ Engl J Med. 2003; 348(17):1681-1691.

5. Lindelof B, Sigurgeirsson B, Gabel H, Stern RS. Incidence of skin cancer in 5356 patients following organ transplantation. Br J Dermatol. 2000; 143(3):513-519.

6. Jensen $P$, et al. Skin cancer in kidney and heart transplant recipients and different long-term immunosuppressive therapy regimens. J Am Acad Dermatol. 1999;40(2 pt 1):177-186.

7. Wiseman DH. Donor cell leukemia: a review. Biol Blood Marrow Transplant. 2011;17(6):771-789.

8. Aractingi $S$, et al. Skin carcinoma arising from donor cells in a kidney transplant recipient. Cancer Res. 2005;65(5):1755-1760.

9. Verneuil L, et al. Human skin carcinoma arising from kidney transplant-derived tumor cells. J Clin Invest. 2013;123(9):3797-3801.

10. Moloney FJ, Comber H, O'Lorcain P, O'Kelly P, Conlon PJ, Murphy GM. A population-based study of skin cancer incidence and prevalence in renal transplant recipients. Br J Dermatol. 2006; 154(3):498-504.

11. Terhorst D, Drecoll U, Stockfleth E, Ulrich C. Organ transplant recipients and skin cancer: assessment of risk factors with focus on sun exposure. Br J Dermatol. 2009;161(suppl 3):85-89.

12. Varley JM. Germline TP53 mutations and Li-Fraumeni syndrome. Hum Mutat. 2003;21(3):313-320.

13. Lapouge $\mathrm{G}$, et al. Identifying the cellular origin of squamous skin tumors. Proc Natl Acad SciUS A. 2011; 108(18):7431-7436.

14. Caulin C, et al. An inducible mouse model for skin cancer reveals distinct roles for gain- and loss-of-function p53 mutations. J Clin Invest. 2007; 117(7):1893-1901.

15. Flynn CM, Kaufman DS. Donor cell leukemia: insight into cancer stem cells and the stem cell niche. Blood. 2007;109(7):2688-2692.

16. Wong G, Chapman JR, Craig JC. Cancer screening in renal transplant recipients: what is the evidence? Clin J Am Soc Nephrol. 2008;3(suppl 2):S87-S100.

17. Snyder TM, Khush KK, Valantine HA, Quake SR. Universal noninvasive detection of solid organ transplant rejection. Proc Natl Acad Sci US A. 2011; 108(15):6229-6234

18. Iznerowicz A, et al. Duration of brain death and cold ischemia time, but not warm ischemia time, increases expression of genes associated with apoptosis in transplanted kidneys from deceased donors. Transplant Proc. 2011;43(8):2887-2890. 


\title{
Factor V, tissue factor pathway inhibitor, and east Texas bleeding disorder
}

\author{
George J. Broze Jr. and Thomas J. Girard
}

Division of Hematology, Washington University School of Medicine, St. Louis, Missouri, USA.

\begin{abstract}
In a report reading like a fascinating detective story, Vincent and colleagues crack the mysterious case of east Texas bleeding disorder. They show that affected individuals have a mutation in exon 13 of the coagulation $F 5$ gene that causes increased expression of an alternatively spliced transcript, which encodes a previously unrecognized factor $V(F V)$ isoform they call $\mathrm{FV}$-short. This FV isoform lacks a large portion of the $\mathrm{B}$ domain of $\mathrm{FV}$, which is normally released upon the proteolytic activation of $\mathrm{FV}$ by thrombin and binds tightly to the coagulation regulator tissue factor pathway inhibitor- $\alpha$ $(\mathrm{TFPI} \alpha)$. This interaction leads to an approximately 10 -fold increase in the level of TFPI $\alpha$ circulating in plasma and a resultant anticoagulant effect that produces a hemorrhagic diathesis.
\end{abstract}

\section{The players: TFPI $\alpha$ and FV}

Full-length tissue factor pathway inhibitor- $\alpha$ (TFPI $\alpha$ ) contains an acidic $\mathrm{N}$ terminus followed by three tandem Kunitztype protease inhibitory domains and a basic $\mathrm{C}$ terminus. It regulates coagulation by producing factor $\mathrm{Xa}$-dependent (FXadependent) feedback inhibition of the factor VIIa/tissue factor complex (FVIIa/TF) (1), which is responsible for the initiation of coagulation, and by directly inhibiting FXa in a process that is enhanced by protein S (PS) $(2,3)$. Full-length TFPI $\alpha$ circulates at a low concentration $(-0.16 \mathrm{nM})$ in plasma, but platelets carry approximately $50 \%$ of total blood TFPI $\alpha$ and release it at sites of injury where they aggregate.

Plasma levels of TFPI $\alpha$ are reduced in patients with factor $V(F V)$ or PS deficiency $(4,5)$. Full-length TFPI $\alpha$ (MW $42 \mathrm{kDa}$ ) circulating in plasma is bound in two high molecular complexes (MW > $700 \mathrm{kDa}$ ) that require the presence of $\mathrm{FV}$ and the basic $\mathrm{C}$ terminus of TFPI $\alpha$, which is needed for its interaction with FV (6). These high molecular weight complexes may contain additional constituents (e.g., PS).

The activated form of FV (FVa) is a coagulation cofactor that dramatically accelerates FXa activation of prothrombin to thrombin. FV is a $330-\mathrm{kDa}$ singlechain protein that circulates in plasma at a concentration of approximately $20 \mathrm{nM}$.

Conflict of interest: The authors have declared that no conflict of interest exists.

Citation for this article: J Clin Invest. 2013; 123(9):3710-3712. doi:10.1172/JCI71220.
About $20 \%$ of the total FV in blood is carried by platelets as fragmented, partially activated forms (7). FV is activated by FXa or thrombin through the proteolytic release of its large, intervening $\mathrm{B}$ domain with the ultimate production of the heavy $(105 \mathrm{kDa})$ and light $(74 \mathrm{kDa})$ chains of FVa that associate in a $\mathrm{Ca}^{2+}$-dependent fashion (Figure 1A).

Elegant studies by Bos and Camire demonstrated that the B domain (aa 710-1545) serves to maintain FV in an inactive, procofactor state and that an interaction between a basic region (BR) (aa 963-1008) and an acidic region (AR) (aa 1493-1537) within the $\mathrm{B}$ domain are required for this effect (Figure 1A and ref. 8). Two peptides within the BR (aa 983-994 and 997-1008) were shown to be most important for its presumed binding to the AR (Figure 1B). Deletion of either the basic or acidic portions of the $\mathrm{B}$ domain produces derivatives of FV with cofactor activity.

\section{The plot}

In 2001, Kuang et al. described a large Texas kindred with a moderately severe bleeding disorder that was characterized by bruising, epistaxis, menorrhagia, and hemorrhage following trauma or surgery that frequently required blood transfusion (9). The prothrombin time (PT) and the activated partial thromboplastin time (aPTT) were both prolonged, suggesting an abnormality in the "common" coagulation pathway that consists of FX, FV, prothrombin, and fibrinogen. Assays of all the coagulation factors, however, produced normal results. Autosomal dominant inheritance and a mild inhibitor pattern in coagulation studies in which patient plasma is mixed with normal plasma suggested a gain-of-function mutation that limited coagulation.

One possible explanation of these results was enhanced inactivation of FXa and thrombin by a hyperactive form of the coagulation inhibitor antithrombin. Initial linkage analysis using an intragenic microsatellite marker showed that the disorder indeed mapped to a locus near the antithrombin gene. Sequencing of the antithrombin gene, however, failed to identify a mutation. More defined linkage studies narrowed the involved locus to a $1.5-\mathrm{Mb}$ region $(1 \mathrm{q} 24)$ that was centromeric to the antithrombin gene and contained the gene for FV (F5) (9). Subsequent sequencing of the $F 5$ gene in an affected individual identified a novel A2440G nucleotide alteration in exon 13 that segregated with the disease and produced a S756G substitution in the $\mathrm{B}$ domain of FV. Since the B domain of FV is not required for FV activity and FV clotting activity in affected family members was normal, the alteration was felt to represent a private polymorphism within the family and unlikely to be associated with the bleeding disorder. Thus, east Texas bleeding disorder remained unexplained.

\section{More detective work}

To determine whether the A2440G alteration in the FV genome detected by Kuang et al. produced an effect on the level or size of the FV protein, Vincent and colleagues (10) analyzed the plasma of family members by Western blotting. They identified a unique $250-\mathrm{kDa}$ band that was prominent in the plasma of affected and barely detectable in the plasma of unaffected family members and confirmed by mass spectrometry that it represented a form of FV. A subsequent search for potential mRNA splicing abnormalities using RT-PCR and mRNA from family members' white blood cells detected the expected $F 5$ transcript ( 2950 bp) and a shorter transcript ( $\sim 840 \mathrm{bp}$, encoding 